\title{
Protective effect against type 2 diabetes mellitus identified within the $A C D C$ gene in a black South African diabetic cohort
}

\author{
Antonel Olckers $^{\mathrm{a}, \mathrm{b}}$, G. Wayne Towers ${ }^{\mathrm{a}}$, Annelize van der Merwe ${ }^{\mathrm{a}, \mathrm{b}}$, Peter E.H. \\ Schwarz $^{c}$, Paul Rheeder ${ }^{d}$ and Aletta E. Schutte ${ }^{e}$ \\ ${ }^{\mathrm{a}}$ Centre for Genome Research, North-West University (Potchefstroom Campus), PO Box \\ 255, Persequor Park, Pretoria 0020, South Africa \\ ${ }^{\mathrm{b}}$ DNAbiotec (Pty) Ltd, Persequor Park, Pretoria 0020, South Africa \\ ${ }^{\mathrm{c}}$ Department of Endocrinopathies and Metabolic Diseases, Medical Faculty Carl-Gustav- \\ Carus, Technical University Dresden, 01307 Dresden, Germany \\ ${ }^{\mathrm{d}}$ Department of Clinical Epidemiology, University of Pretoria, Pretoria 0001, South \\ Africa \\ ${ }^{\mathrm{e}}$ School for Physiology, Nutrition and Consumer Sciences, North-West University \\ (Potchefstroom Campus), Potchefstroom 2520, South Africa
}

\begin{abstract}
Type 2 diabetes mellitus (T2D) is currently one of the fastest growing noncommunicable diseases in the world. It is induced by the pathogenic interaction between insulin resistance and secretion. This group of clinically heterogeneous disorders currently affects approximately $4 \%$ of the general population, but it is rapidly increasing, especially in developing regions such as sub-Saharan Africa. During this investigation, a diabetic (n $=227)$ and control cohort $(\mathrm{n}=226)$ of adult black South African individuals were screened for the reported single nucleotide polymorphisms, termed C-11377G and G$11391 \mathrm{~A}$, within the promoter of the adiponectin $(A C D C)$ gene. Genotyping was achieved via a real-time polymerase chain reaction method. It was determined that the variant allele at G-11391A as well as the 12 haplotype was significantly associated with a
\end{abstract}


protective factor with regard to T2D susceptibility. The low frequency of this variant within the cohorts investigated indicated a minor role in decreasing disease susceptibility. It may not be a significant disease risk factor in itself, but may assist in elucidating the mechanism of disease susceptibility. When compared to various non-African populations, it becomes apparent that the investigated single nucleotide polymorphisms have differential effects depending on the population investigated. This investigation therefore underscores the genetic heterogeneity at T2D susceptibility loci within the black South African population.

\section{Article Outline}

1. Introduction

2. Research design and methods

2.1. Study design and patient selection

2.2. Genotype determination

2.3. Statistical and clinical analyses

3. Results

3.1. Analysis of $A C D C$ SNPs

3.2. Haplotype analysis

3.3. Association between genetic and biochemical factors

4. Discussion

Acknowledgements

References

\section{Introduction}

Complex disorders present due to numerous interactions among environmental, genetic, and endogenous factors [1] and [2]. Elucidation of the effects that each of these separate entities have on the disease phenotype is therefore central to our understanding of the pathogenesis of these disorders. Type 2 diabetes mellitus (T2D) is currently one of the 
most prominent complex disorders due to its high frequency of $4 \%$ in the global population, with a predicted prevalence of $5.4 \%$ by the year 2025 [3]. The largest predicted increase, estimated at $185 \%$, will occur within developing countries, of which 5 million will be in sub-Saharan Africa alone [3].

This group of disorders is highly heterogeneous, but all forms are characterized by chronic hyperglycemia and variations in cellular metabolism. The initial dysregulation in T2D is at the level of insulin resistance, which increases insulin secretion failure, culminating in the disease phenotype [4] and [5]. T2D is thus induced by the pathogenic interaction between insulin resistance and secretion. Although this is a classic example of a complex disorder, T2D does present with a strong familial association, and it is therefore imperative to dissect the genetic components of this group of diseases.

In the investigation of quantitative trait loci involved in the susceptibility toward the metabolic syndrome, it was determined that a locus at $3 \mathrm{q} 27$ was strongly linked to the following 6 traits that are fundamental to the expression of this cluster of disorders [6]: body mass index (BMI), waist circumference, fasting plasma insulin, body weight, hip circumference, and the glucose-insulin ratio. The gene encoding adiponectin was subsequently identified at this locus [7], and in an investigation by Weyer et al [8], it was determined that hypoadiponectinemia was significantly associated with increased risk for T2D, whereas hyperadiponectinemia was associated with increased protection against developing these disorders.

Adiponectin has both a glucose-sensitizing effect as well as the ability to increase the fatty acid-oxidizing capacity of the cell. Adiponectin further exerts its antidiabetic effects by decreasing glucose production in the liver [9]. The association of hypoadiponectinemia with insulin resistance and hyperinsulinemia described by Weyer et al [8] was probably caused by a defect in the aforementioned pathway. Any alteration on a genetic level that affects the production of this protein, thus leading to impaired insulin action, may consequently result in $\mathrm{T} 2 \mathrm{D}$. 
Various single nucleotide polymorphisms (SNPs) within the adiponectin gene have been associated with T2D disease risk as well as increased BMI [10], [11] and [12]. The SNPs may induce their effects by affecting the binding of transcription factors and disrupting regulatory elements, whereas the missense alterations may affect or disrupt the formation of specific isoforms as well as the higher structure of this protein. In an investigation by Vasseur et al [11], it was determined that 2 promoter SNPs, C-11377G and G-11391A, within the adiponectin gene were associated with increased risk for T2D within the French population. The means by which these alterations affect disease susceptibility remains unclear [11]. It was further determined that a haplotype containing the variant allele at C-11377G and the wild-type allele at G-11391A (21 haplotype) was associated with a significantly elevated diabetes risk. In a similar analysis, the 12 haplotype was in turn associated with T2D risk in the German Caucasian population [13]. Analysis of a Japanese cohort did not result in any significant association with these 2 loci, with an absence of homozygotes for the variant allele at G-11391A within this group [14]. Alterations in the adiponectin gene are associated with T2D risk and increased obesity. The relevance of family history to T2D risk has been highlighted in the black South African population; however, very little is known with regard to the specific genetic factors at play [15]. We determined that the majority of persons with diabetes in the African population tend to present with high BMI values, leading to the hypothesis that the $A C D C$ gene may play a significant role. The black African population belongs to macrohaplogroup L, which harbors the most ancient human lineage and has the highest level of variation [16]. The high level of genetic variation may be synchronized with the clinical heterogeneity observed in the African diabetic population. This African population is ancestral in nature, and all other modern populations arose from migrations of this group out of Africa [17]. Our investigation was undertaken to determine if the genetic structures within the $A C D C$ gene that are associated with disease risk in the Caucasian population were similarly associated in the African population. 


\section{Research design and methods}

This research program has been approved by the Ethics Committee of the North-West University (Potchefstroom Campus). Written informed consent was obtained from all subjects before their inclusion in the project.

\subsection{Study design and patient selection}

Four hundred fifty-three black South African individuals were included in this investigation. Individuals were from linguistically heterogeneous backgrounds. However, because of the current paucity of data linking linguistic background to genetic ancestry in the black South African population, the current cohorts were not stratified according to their current language preference. The cohorts investigated consisted mainly of individuals with Tswana and Northern Sotho ethnic origins.

The patient $(n=227)$ and control $(n=226)$ cohorts were collected from various diabetic and outpatient clinics within the Gauteng and Northwest provinces of South Africa. All patients were diagnosed via the criteria stipulated by the World Health Organization consortium [5] through verification of individual patient records or clinical investigation. Controls were recruited from similar clinical locations as the patients. Furthermore, these individuals were collected from clinics that screen for metabolic diseases, eg, hypertension clinics, thus ensuring no undiagnosed diabetic patients would be included. A random glucose reading was also obtained for each control individual at the initial outset of the consultation, and they were confirmed to be nondiabetic if the reading was less than $7 \mathrm{mmol} / \mathrm{L}$.

A subset of 100 unaffected black South African women each underwent a 2-hour oral glucose tolerance test after an overnight fasting period of 10 to 12 hours. A fasting sample was drawn after which a glucose load ( $75 \mathrm{~g}$ glucose in $300 \mathrm{~mL}$ water) was given to the individual to drink. Samples were again collected at 30, 60, 90, and 120 minutes after the glucose load. Whole blood was appropriately collected in serum gel tubes (for analysis of adiponectin) and fluoride tubes (for analysis of plasma glucose) followed by 
centrifugation at $3000 \mathrm{rpm}$ for 5 minutes. The supernatant was aliquoted and stored at $-70^{\circ} \mathrm{C}$ until used.

These individuals were analyzed for fasting adiponectin levels as well as plasma glucose at all 5 time intervals. Adiponectin levels at fasting were determined via the use of the Human Adiponectin ELISA Kit (BioCat, Heidelberg, Germany), whereas plasma glucose was measured by using a hexokinase-based method.

\subsection{Genotype determination}

DNA was isolated from whole blood collected in EDTA tubes. Genetic screening was achieved via the use of a real-time polymerase chain reaction (PCR) strategy using the LightCycler (LC) Real-Time PCR machine (Roche Molecular Biochemicals, Indianapolis, IN). Genotypes were obtained via the use of hybridization probes and melt curve analyses. The LC reaction consisted of the following components: 10 pmol of each of the forward and reverse primers (see Table 1); 3 pmol of the anchor probe (Table 1); $1.5 \mathrm{pmol}$ of the sensor probe (Table 1); and $3.75 \mu \mathrm{mol} / \mathrm{L} \mathrm{MgCl}_{2}$ and $2 \mu \mathrm{L}$ of the FastStart Reaction Mix Hybridization Probes $(10 \times$ reaction buffer) containing the LC-FastStart enzyme. Amplification was achieved via the following thermal cycling conditions: initial denaturation at $95^{\circ} \mathrm{C}$ for 10 minutes was followed by 36 cycles consisting of a denaturation step at $95^{\circ} \mathrm{C}$ for 10 seconds, an annealing step at $58^{\circ} \mathrm{C}$ for 7 seconds, and an elongation step at $72^{\circ} \mathrm{C}$ for 9 seconds. 


\section{Table 1.}

Primers and hybridization probes used in the detection of specific SNPs within the $A C D C$ gene

\begin{tabular}{|l|l|l|}
\hline SNP & Primer & Primer sequence \\
\hline C-11377G & ACR1F & Forward: $5^{\prime}$-act tgc cct gcc tct gtc tg-3' \\
\hline G-11391A & ACR1R & Reverse: $5^{\prime}$-gcc tgg aga act gga agc tg-3' \\
\hline
\end{tabular}

\begin{tabular}{|l|l|l|}
\hline SNP & Probe & Probe sequence \\
\hline C-11377G & SNP1 anchor & 5'-LC 640-aca tga gcg tgc caa gaa agt cca agg tgt tg-p-3' \\
\hline G-11391A & AnchorAPM10-1 & $\begin{array}{l}\text { 5'-LC640-gca agc cac aca ttc tga tga att aaa tta cga ccc-p- } \\
3^{\prime}\end{array}$ \\
\hline & SensorGsnp10 & $5^{\prime}$-gca gga tct gag ccg gtt ct-X-3' \\
\hline
\end{tabular}

X indicates fluorescein; LC640, LightCycler640 red fluorophore; p, phosphorylated 3' end.

Melt curve analysis was achieved by decreasing the temperature to $30^{\circ} \mathrm{C}$ and subsequently increasing it by $0.5^{\circ} \mathrm{C}$ per second up to a final temperature of $90^{\circ} \mathrm{C}$. Fluorescence was detected continuously throughout this process and was plotted graphically as fluorescence vs temperature by the LightCycler software. The melting peak of each SNP was obtained via determination of the negative derivative of the fluorescence and plotted against temperature $(-\mathrm{d} F / \mathrm{d} T$ vs $T)$.

\subsection{Statistical and clinical analyses}

Unless otherwise stated, all statistical analyses were undertaken by using Statistica version 7.0 software (Statsoft Inc, Tulsa, OK) [18]. Continuous clinical data are expressed as the mean $\pm \mathrm{SD}$. Association of a specific genotype at the previously 
described loci was achieved by determining whether the populations under investigation adhered to Hardy-Weinberg equilibrium. After ascertainment of adherence to HardyWeinberg proportions, haplotypes were inferred by the excitation-maximization method via the use of the Arlequin version 3.01 software (Laurent Excoffier, Bern, Switzerland) [19]. Subsequently, comparison of the genotype and haplotype frequencies between the patient and control cohorts was achieved by using contingency table analyses,

specifically $\chi^{2}$ testing (for $3 \times 2$ contingency tables) and Fisher exact test (for tables with zero cells). Odds ratio (OR) was calculated via Woolf's method by using the combined counts of the genotypes not undergoing analysis as the reference group [20].

Within the subset of the 100 individuals that underwent oral glucose tolerance tests, biochemical data were tested for adherence to the normal distribution by using the Shapiro-Wilk $W$ test. Nonnormal data were analyzed for global effects via the KruskalWallis analysis of variance (ANOVA) test after which specific differences in different genotype groups at each locus were determined via multiple comparison of ranks. Normally distributed data were analyzed for a global difference via 1-way ANOVA, whereas specific differences between genotype classes were analyzed by the application of the Tukey unequal $N$ Honestly Significant Difference test.

\section{Results}

\subsection{Analysis of $A C D C$ SNPs}

During this investigation, a diabetic cohort $(\mathrm{n}=227)$ and a control cohort $(\mathrm{n}=226)$ of adult black South African individuals were screened for 2 reported SNPs within the promoter region of the $A C D C$ gene. The clinical data of the cohorts are presented in Table 2. As presented, the BMI and waist-hip ratio are very similar in both groups, thus decreasing the likelihood of variations within these clinical factors masking any of the genetic associations determined. Genotype frequencies at both loci were consistent with the expected distribution of genotypes in a population undergoing Hardy-Weinberg equilibrium in all cohorts investigated. It was thus possible to determine if any significant alterations in the frequencies of the various genotypes were present between the affected and unaffected cohorts. It was determined via Fisher exact test, as presented in Table 3, 
that the heterozygous genotype at G-11391A was associated with protection toward T2D (OR, $0.15 ; 95 \%$ confidence interval [CI], 0.03-0.71; $P=.0065$ ).

\section{Table 2.}

Clinical data of the individuals included in the study (mean \pm SD)

\begin{tabular}{|l|l|l|}
\hline Clinical indicator & Patient cohort & Control cohort \\
\hline $\mathrm{n}($ female/male) & $196 / 31$ & $195 / 31$ \\
\hline Age (y) & $57 \pm 11.6$ & $44 \pm 17.3$ \\
\hline BMI (kg/m ${ }^{2}$ ) & $31.5 \pm 8.5$ & $31.6 \pm 7.3$ \\
\hline Waist-hip ratio & $0.9 \pm 0.1$ & $0.9 \pm 0.1$ \\
\hline Random glucose (mmol/l) & $11.0 \pm 6.4$ & $5.0 \pm 0.9$ \\
\hline Age at diagnosis (y) & $49 \pm 13.1$ & NA \\
\hline
\end{tabular}

$\mathrm{n}$ indicates individuals for whom genotype data was generated at any of the SNP loci;

NA, not applicable.

\section{Table 3.}

$\chi^{2}$ Analysis for the comparison of the genotype distribution between the black South African patient and control cohorts

\begin{tabular}{|l|l|l|l|l|}
\hline SNP & \multicolumn{2}{|l|}{ Genotypes } & $P$ \\
\hline C-11377G & 1.1 & 1.2 & 2.2 & $<.19$ \\
\hline Patient & $0.73(166)$ & $0.26(60)$ & $0.00(1)$ & \\
\hline Control & $0.71(161)$ & $0.27(60)$ & $0.02(5)$ & \\
\hline OR (95\% CI) & $1.09(0.72-1.65)$ & $0.99(0.65-1.50)$ & $0.19(0.02-1.68)$ & \\
\hline G-11391A & 1.1 & 1.2 & - & .0065 \\
\hline Patient & $0.99(225)$ & $0.01(2)$ & & \\
\hline Control & $0.95(214)$ & $0.05(12)$ & & \\
\hline OR (95\% CI) & $6.30(1.39-28.5)$ & $0.15(0.03-0.71)$ & & \\
\hline
\end{tabular}




\subsection{Haplotype analysis}

Haplotypes were inferred by using the excitation-maximization method of the Arlequin version 3.0 software with an epsilon value of $1 \times 10^{-7}$. To ensure the global maximum likelihood was calculated, 50 independent starting points were implemented with a maximum number of 1000 iterations. The relative haplotype values were transformed into absolute counts that were compared with each other within the patient and control cohorts (see Table 4). The 12 haplotype was significantly associated with a protective effect against T2D (OR, 0.16; 95\% CI, 0.03-0.72; $P<.01)$. However, this alteration is at a relatively low frequency within this cohort, indicating that it may not have a significant effect on a population level, but instead affords protection on the individual level. The identification of factors that act at the population level will require analysis of a larger cohort.

\section{Table 4.}

$\chi^{2}$ Analysis for the comparison of the haplotype distribution between the black South African patient and control cohorts

\begin{tabular}{|l|l|l|l|l|}
\hline Haplotypes & $\begin{array}{l}\text { Patients, } \\
\text { frequency (n) }\end{array}$ & $\begin{array}{l}\text { Controls, } \\
\text { frequency (n) }\end{array}$ & $\chi^{2}$ & OR (95\% CI) \\
\hline 11 & $0.86(390)$ & $0.82(370)$ & 0.91 & $1.35(0.94-1.92)$ \\
\hline 12 & $0.00(2)$ & $0.03(12)$ & 8.38 & $0.16(0.03-0.72)$ \\
\hline 21 & $0.14(62)$ & $0.15(70)$ & 0.98 & $0.86(0.59-1.24)$ \\
\hline Total & 454 & 452 & 10.27 & - \\
\hline
\end{tabular}

Constitution of haplotype $=\mathrm{C}-11377 \mathrm{G}$ and G-11391A. $\mathrm{n}$ indicates individuals from whom haplotype data, reflecting genotypes at both loci, were generated.

\subsection{Association between genetic and biochemical factors}

Both the fasting adiponectin levels $(P=.00$; median, 9.80; interquartile range, 6.40) as well as the plasma glucose levels $(P=.00$ at $0,30,60,90$, and 120 minutes $)$ at each time interval were determined not to follow a normal distribution. No association was determined between the fasting adiponectin levels and the genotype classes at the $\mathrm{C}$ - 
$11377 \mathrm{G}$ (Kruskal-Wallis $P=.61$ ) and G-11391A (Kruskal-Wallis $P=.48$ ) loci. Analysis of plasma glucose at all 5 time intervals was also not significantly different for the different genotypes present at the above 2 loci as presented in Table 5. Comparison of the haplotypes present within this subset furthermore resulted in no significant association with either the plasma glucose levels (see Table 5) at the different time intervals or the fasting adiponectin values (Kruskal-Wallis $P=.88$ ). This is relevant, as the 21 haplotype within a Caucasian French population was associated with decreased adiponectin levels.

\section{Table 5.}

Kruskal-Wallis ANOVA for the comparison between plasma glucose levels and the genotype and haplotype status at the two $A C D C$ loci under investigation in the subset of 100 black South African females

\begin{tabular}{|l|l|l|l|}
\hline Time (min) & \multicolumn{4}{ll}{ Kruskal-Wallis $P$} \\
\hline & C-11371G & G-11391A & Haplotypes \\
\hline 9 & .19 & .37 & .06 \\
\hline 30 & .70 & .16 & .35 \\
\hline 60 & .55 & .45 & .89 \\
\hline 90 & .22 & .30 & .72 \\
\hline 120 & .17 & .33 & .51 \\
\hline
\end{tabular}

\section{Discussion}

This report represents the largest investigation into the association of certain SNPs within the adiponectin gene with T2D in the black South African population. The wild-type homozygote at the G-11391A locus has an OR greater than 1, which indicates association with disease risk. However, the wide range of the $95 \%$ CI means that care must be taken in any conclusions determined from this result. Alternatively, the heterozygote at G- 
11391A presented with significant association to a protective effect against T2D. However, the relative rarity of this variant allele is surprising, as it seems intuitive to expect that when an alteration is associated with a protective factor it should be preferentially selected. As it is within a late-onset disease susceptibility locus, however, the effect of possible adaptive forces is countered, thus preventing enrichment of this allele [21].

French Caucasian diabetic cohorts harbor the variant alleles at these loci at frequencies of 0.29 and 0.1 at the C-11377G and G-11391A loci, respectively [11]. As depicted in Table 3 , our diabetic cohort harbored these alterations at frequencies of 0.13 and 0.01 , respectively, thus indicating the comparative absence of the variant alleles from the black South African population.

It is possible for certain haplotypes within a gene to be in association with T2D even if the association of the individual genotypes are not significant [22], [23] and [24]. Investigation into the haplotype structure of these black South African individuals was undertaken to determine if this was also true for the $A C D C$ gene. In the French Caucasian population, the 21 haplotype is significantly associated with disease risk for T2D as well as hypoadiponectinemia [11]. This is, however, not the case within our black South African cohort.

It was determined that the 12 haplotype (frequency, 0.03) represents possible association with a protective factor for T2D within the black South African population. This is not surprising, as the variant allele at G-11391A by itself was determined to be associated with such an effect. That this association is maintained at the haplotype level only strengthens the protective role hypothesized for this variant.

The apparent lack of association between the different genotypes and haplotypes discussed and the adiponectin and plasma glucose levels investigated may seem surprising; however, this strengthens the argument that the mechanism of T2D risk in black South Africans is different from that in Caucasians. In the French population 
investigated by Vasseur et al [11], it was determined that the 21 haplotype was associated with decreased adiponectin levels. However, in the black South African population, it is possible that this haplotype is associated with alternative biochemical factors that are important in the signaling functions of adiponectin. Thus, it will be important in the future to investigate the functionality associated with these polymorphisms. Comparison of our African data with that of the German population [13] previously discussed highlighted the fact that the 12 haplotype was associated with T2D susceptibility only in the European population. That the 12 haplotype is associated with disease risk in the German cohort, whereas it may be a protective factor in the black South African population, indicates a differential metabolic effect for genetic alterations in the $A C D C$ gene with regard to T2D risk in these 2 populations. It is possible that the relative absence of variant alleles within the black South African diabetic cohort may be because the 12 haplotype underwent a change in selective pressure on migration into Europe, thus resulting in the disease risk with which it is currently associated.

We suggest a different role for $A C D C$ in $\mathrm{T} 2 \mathrm{D}$ disease susceptibility within the black South African population. Our results do not exclude the entire $A C D C$ gene from playing a role in T2D susceptibility in this population. It may be that various other genetic lesions within the gene itself, which are not in linkage disequilibrium with the alterations investigated, are involved. Our results do, however, allude to the unfortunate fact that intervention strategies developed elsewhere may ultimately not be as effective in our population, as different population-specific risk factors are at play. However, these genetic factors may be effective to stratify personal T2D risk in preventative management. 


\section{References}

[1] A.H. Barnett, C. Eff, R.D.G. Leslie and D.A. Pyke, Diabetes in identical twins: a study of 200 pairs, Diabetologia 20 (1981), pp. 87-93.

[2] R.N. Bergman, L.S. Phillips and C. Cobelli, Physiologic evaluation of factors controlling glucose tolerance in man: measurement of insulin sensitivity and beta-cell glucose sensitivity from the response to intravenous glucose, $J$ Clin Invest 68 (1981), pp. $1456-1467$.

[3] H. King, R.E. Aubert and W.H. Herman, Global burden of diabetes, 1995-2025: prevalence, numerical estimates, and projections, Diabetes Care 21 (1998), pp. 14141431.

[4] World Health Organisation, Handbook of resolutions and decisions of the World Health Assembly and the Executive Board. Vol. III, 1985-1992, WHO, Geneva (1993), pp. 131-132.

[5] K.G.M.M. Alberti and P.Z. Zimmet, World Health Organisation definition, diagnosis and classification of diabetes mellitus and its complications. Report of a WHO consultation, Part 1: diagnosis and classification of diabetes mellitus, WHO Department of Noncommunicable Disease Surveillance, Geneva (1999).

[6] A.H. Kissebah, G.E. Sonnenberg and J. Myklebust et al., Quantitative trait loci on chromosomes 3 and 17 influence phenotypes of the metabolic syndrome, Proc Natl Acad Sci U S A 97 (2000), pp. 14478-14483.

[7] K. Saito, T. Tobe and M. Yoda et al., Regulation of gelatin-binding protein 28 (GBP28) gene expression by C/EBP, Biol Pharm Bull 22 (1999), pp. 1158-1162.

[8] C. Weyer, T. Funahashi and S. Tanaka et al., Hypoadiponectinemia in obesity and type 2 diabetes: close association with insulin resistance and hyperinsulinemia, $J$ Clin Endocrinol Metab 86 (2001), pp. 1930-1935.

[9] T. Yamauchi, J. Kamon and H. Waki et al., The fat-derived hormone adiponectin reverses insulin resistance associated with both lipoatrophy and obesity, Nat Med 7 (2001), pp. 941-946.

[10] M. Stumvoll, O. Tschritter and A. Fritsche et al., Association of the T-G polymorphism in adiponectin (exon 2) with obesity and insulin sensitivity, Diabetes $\mathbf{5 1}$ (2002), pp. 37-41. 
[11] F. Vasseur, N. Helbecque and C. Dina et al., Single-nucleotide polymorphism haplotypes in both the proximal promoter and exon 3 of the APM1 gene modulate adipocyte-secreted adiponectin hormone levels and contribute to the genetic risk for type 2 diabetes in French Caucasians, Hum Mol Genet 11 (2002), pp. 2607-2614.

[12] H. Kondo, I. Shimomura and Y. Matsukawa et al., Association of adiponectin mutation with type 2 diabetes, Diabetes 51 (2002), pp. 2325-2328.

[13] P.E.H. Schwarz, S. Govindarajalu, W. Towers, U. Schwanebeck, S. Fischer and F. Vasseur et al., Haplotypes in the promoter region of the $A D I P O Q$ gene are associated with increased diabetes risk in a German Caucasian population, Hormone Metab Res 38 (2006), pp. 447-454.

[14] K. Hara, P. Boutin and Y. Mori et al., Genetic variation in the gene encoding adiponectin is associated with an increased risk of type 2 diabetes in the Japanese population, Diabetes 51 (2002), pp. 536-540.

[15] R.T. Erasmus, E. Blanco Blanco and A.B. Okesina et al., Importance of family history in type 2 black South African diabetic patients, Postgrad Med J 77 (2001), pp. 323-325.

[16] Y.-S. Chen, A. Torroni and L. Excoffier et al., Analysis of mtDNA variation in African populations reveals the most ancient of all human continent-specific haplogroups, Am J Hum Genet 57 (1995), pp. 133-149.

[17] R.L. Cann, M. Stoneking and A.C. Wilson, Mitochondrial DNA and human evolution, Nature 325 (1987), pp. 31-36.

[18] StatSoft Inc, STATISTICA (data analysis software system), version 7www.statsoft.com (2004).

[19] Excoffier LGL, Schneider S. Arlequin ver. 3.0: an integrated software package for population genetics data analysis. Evolutionary Bioinformatics Online, submitted 2005. [20] J.M. Bland and D.G. Altman, The odds ratio, Br Med J 320 (2000), p. 1468. [21] A. Wright, B. Charlesworth and I. Rudan et al., A polygenic basis for late-onset disease, Trends Genet 19 (2003), pp. 97-106.

[22] Y. Horikawa, N. Oda and N.J. Cox et al., Genetic variation in the gene encoding calpain-10 is associated with type 2 diabetes mellitus, Nat Genet 26 (2000), pp. 163-175. 
[23] P.G. Cassell, A.E. Jackson and B.V. North et al., Haplotype combinations of calpain 10 gene polymorphisms associate with increased risk of impaired glucose tolerance and type 2 diabetes in South Indians, Diabetes 51 (2002), pp. 622-628.

[24] M.T. Malecki, D.K. Moczulski and T. Klupa et al., Homozygous combination of calpain 10 gene haplotypes is associated with type 2 diabetes mellitus in a Polish population, Eur J Endocrinol 146 (2002), pp. 695-699.

Corresponding author. Professor Antonel Olckers, Director: Centre for Genome Research, Centre for Genome Research, North-West University (Potchefstroom Campus), PO Box 255, Persequor Park, Pretoria, 0020, South Africa. Tel.: +27 12349 1685; fax: +27 123491689 . 